bacterial metabolism. We aimed to compare the faecal metabolome before and after ferrous sulphate supplementation.

Methods 77 faecal samples were collected from patients with iron deficiency anaemia, before treatment and after two months of therapy. Faecal headspace gases were analysed using gas chromatography-mass spectrometry: VOC identification involved matching mass spectra against the NIST Library. Univariate and multivariate analysis was performed on the VOCs found, including partial least squares regression (PLS-DA).

Results A significant change in abundance of 17 VOCs was found. Adjustment was made for the number of comparisons: one VOC was then shown to increase significantly. The median abundance of 2-pentylfuran changed four-fold (FDR adjusted $P=0.006$ ) in patients taking ferrous sulphate for two months (Fig. 1A). Overall, a plot to illustrate the PLS-DA shows how the pre- and post-treatment samples differ (Fig. 1B). Though the abundance changed in 16 other VOCs-which included aldehydes, esters and ketones-their significance was lost after correction for multiple testing, which indicates that the study may be underpowered.

Conclusions The abundance of faecal 2-pentylfuran increases significantly during ferrous sulphate therapy. 2-pentylfuran is a metabolite of fungi. It remains to be seen whether ferrous sulphate directly acts on fungi or whether there is an interaction between iron and bacteria, and then between bacteria and fungi. It is very clear that faecal metabolites are influenced by ferrous sulphate supplementation.

\section{OWE-22 ROLE OF LOW GRADE DYSPLASIA SMALL POLYPS DURING SCREENING COLONOSCOPIES SURVEILLANCE: A PROSPECTIVE COHORT STUDY}

${ }^{1}$ Diego Caroli*, ${ }^{1}$ Erik Rosa-Rizzotto, ${ }^{1}$ Mario Saia, ${ }^{2}$ Ennio Guido, ${ }^{1}$ Manuel Zorzi, ${ }^{2}$ Franca De Lazzeri. ${ }^{1}$ St. Anthony Hospital, Padova, Italy; ${ }^{2}$ Azienda Zero, Padova, Italy

\subsection{6/gutjnl-2019-BSGAbstracts.355}

Background The role diminutive polyps and small polyps play in the development of advanced neoplasia (AN) or cancer during follow-up (FU) continues to be debated. Recent studies have shown that the risk of AN development during $\mathrm{FU}$ is higher in patients with $>5$ small polyps but similar in patients with - or - small polyps ${ }^{2,3}$. Given these findings, the current study aimed to evaluate if current European FU guidelines ${ }^{1}$ at first screening colonoscopy according to the number of low-grade dysplasia tubular adenoma $<1$ cm ("micropolyps”, MP) are too strict.

Methods A longitudinal cohort study was carried out on a representative sample $(50 \%)$ of patients who underwent a screening colonoscopy (clean colon) in 2010 showing at least 1 polyp. Patients with AN or cancers were excluded. Polyps $>1 \mathrm{~cm}$ or villous or high grade dysplasia/carcinoma in situ were considered AN. These patients made a FU colonscopy according to UE screening CCR guidelines. Patients were split up according to European guidelines in Low risk (- MP), Intermediate risk ( $-\mathrm{MP})$ and High risk (>5 MP). Data were analyzed by SPSS program.

Results Of 640 patients included in the sample 172 (27\%) were included in the surveillance program (mean age 62,2 \pm 5,7 yr, 5-0), 120 male (69,8\%). During first colonoscopy (2010) 370 MP were detected $(M \pm S D ~ 2,1 \pm 1,5$, range -3 polyps for each patient). During FU (median 5.5 years), 315 colonoscopies were performed (mean 1.8; range -) and an
AN was detected in 23 patients (13.4\%): 20/23 tubulovillous microadenoma, 1/23 high grade dysplasia, 2/23 tubular adenoma $>1 \mathrm{~cm}$. The detection of AN among patients in treatment with ASA was non significantly lower than the others. AN detection was lower among Low risk patients (13/ $108 ; 10.7 \%)$ as compared to Intermediate risk $(7 / 33 ; 17.5 \%)$ and High risk $(3 / 8 ; 27.3 \%) \quad(\mathrm{P}=\mathrm{ns})$. According to KaplanMeier analysis, the cumulative risk of $\mathrm{AN}$ among High risk was significantly increased $(p=0.035)$ (figure 1$)$. No interval cancers were found during the follow-up.

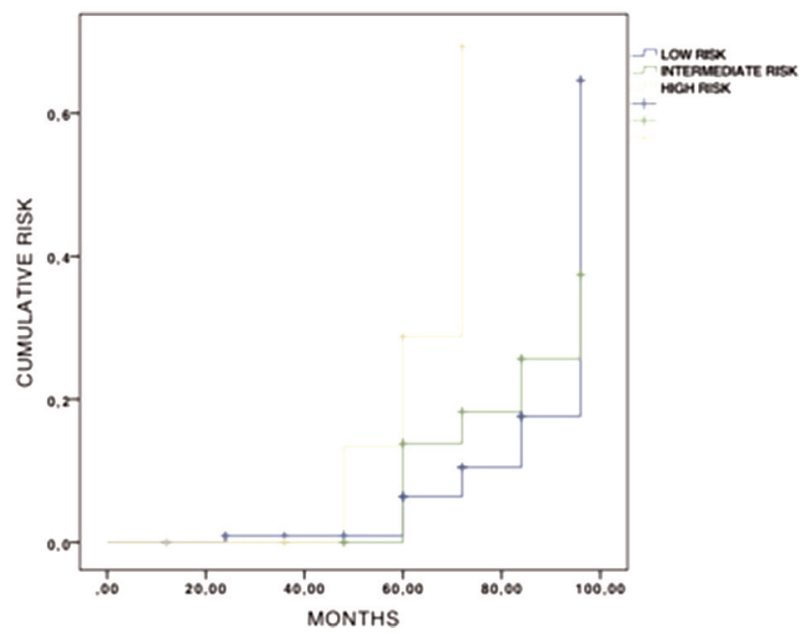

Abstract OWE-22 Figure 1

Conclusions These findings suggest that EU guidelines for surveillance colonoscopies for $>3$ small LGD polyps are excessively strict. We propose extending the time for a repeat colonscopy FU for these patients to $5 \mathrm{yrs}$

\section{REFERENCES}

1. Segnan N. et al. European Guidelines for Quality Assurance in Colorectal Cancer Screening and Diagnosis First Edition 2011

2. Chang MM, et al. Digest Liv Dis 2018; 50: 847-852

3. Jung YK, et al. Am J Gastroenterol 2018: 113(12):185-1.

\section{OWE-23 VERSATILE ROLE OF SECRETED FRIZZLED RELATED PROTEIN 2 (SFRP2) IN COLON CANCER: POTENTIAL STROMAL TARGET}

${ }^{1,2}$ Maria Tabuso* ${ }^{2}$ Raghu Adya, ${ }^{1}$ Kishore Gopalakrishnan, ${ }^{1}$ Sean James, ${ }^{1}$ Andrew White,
${ }^{1}$ Adrian Fisk, ${ }^{2}$ Federica Dimitri, ${ }^{2}$ Jennifer Butler, ${ }^{2}$ Mark Christian, ${ }^{1,2}$ Ramesh P Arasaradnam.
${ }^{1}$ UHCW, Coventry, UK; ${ }^{2}$ University of Warwick, Warwick, UK

\subsection{6/gutjnl-2019-BSGAbstracts.356}

Introduction Dysfunctional adipose tissue has emerged as a key contributor to colorectal cancer. However, the role of peritumoral adipose tissue (pAT) in colon cancer has not been widely investigated. Aim of this study is to identify differentially expressed genes (DEGs) associated with cancer pathways in peritumoral adipose tissue compared with adjacent normal adipose tissue in human colon cancer.

Methods Fresh peritumoral and distal adipose tissue samples were collected from 6 patients undergoing surgery for colon cancer stage T3/T4. DEGs were identified employing Nanostring PanCancer Pathway Panel including 770 cancer-associated genes. Criteria for differential expression included p-value 